\title{
AN EXISTENCE THEOREM IN THE ALGEBRAIC STUDY OF HOMOGENEOUS LINEAR ORDINARY DIFFERENTIAL EQUATIONS
}

\author{
MARVIN P. EPSTEIN ${ }^{1}$
}

Introduction. The Picard-Vessiot theory is a Galois theory of homogeneous linear ordinary differential equations. Unlike ordinary Galois theory, however, in which fixing a universe automatically determines the zeros of a given polynomial, Picard-Vessiot theory is complicated by the fact that a given homogeneous linear ordinary differential polynomial $L(y)$ of order $\geqq 1$ possesses infinitely many zeros. To simplify matters, therefore, one wishes to choose those zeros of $L(y)$ which are the "simplest" in a certain sense. More specifically, it is seen in a paper (to appear) generalizing the PicardVessiot theory as presented by Kolchin [1] that the algebraic and topological structures involved in that generalization are strongly dependent on the constants introduced when one adjoins zeros of $L(y)$ to the ground field. It is desirable, therefore, to choose zeros of $L(y)$ in such a manner that the constants introduced by these zeros are subject to certain restrictive conditions. This requires a theorem asserting the existence of zeros of $L(y)$ for which these restrictive conditions are satisfied. The main result of this paper is a theorem on the existence of this special type of zeros of $L(y)$.

We fix an abstract ordinary differential field $F$ of characteristic zero and with field of constants $C$ (i.e. $F$ is an abstract field of characteristic zero in which a derivation is defined, and $C$ is the set of all elements of $F$ which have derivative equal to 0 ). We shall assume throughout that we are given $\Omega$, a universal differential extension field of $F$, the existence of which is proved in [3, Chap. I, §5]. This universal extension will contain all the elements which enter the discussion. In particular, $\Omega$ will contain an algebraic closure $\bar{C}$ of $C$.

We use the standard notation for derivatives; thus, for $\alpha \in \Omega$ the successive derivatives of $\alpha$ are denoted $\alpha^{\prime}, \alpha^{\prime \prime}, \cdots, \alpha^{(r)}, \cdots$. For the adjunction of elements to differential fields (for example, to the differential field $F$ ), we shall use $F(\cdots)$ to denote ordinary field adjunction, $F\{\cdots\}$ to denote differential ring adjunction, and $F\langle\cdots\rangle$ to denote differential field adjunction: thus, for example,

Received by the editors March 12, 1954.

1 This paper is part of a Doctoral Dissertation written at Columbia University under the direction of E. R. Kolchin. The research was performed under the sponsorship of the Atomic Energy Commission. 
$F\langle\alpha, \beta\rangle=F\left(\alpha, \beta, \alpha^{\prime}, \beta^{\prime}, \cdots\right)$. Also the symbol $\{\cdots\}$ will stand for the perfect differential ideal generated by the elements therein (i.e. the minimal radical ideal which contains these elements and which is closed under differentiation).

The letters $X, t, y$ (with or without subscripts) will denote indeterminates. We shall say that an element $\alpha \in \Omega$ is a nontrivial zero of a differential polynomial $P(y)$ if $\alpha \neq 0$ but $P(\alpha)=0$.

By $L(y)$ we mean a fixed homogeneous linear ordinary differential polynomial

$$
L(y)=y^{(n)}+p_{1} y^{(n-1)}+\cdots+p_{n} y \quad\left(n \geqq 1, \text { each } p_{i} \in F\right) .
$$

A system $\left(\eta_{1}, \cdots, \eta_{n}\right)$ of zeros, of $L(y)$ is called fundamental if the system is linearly independent over constants; it will be called regular (with respect to $F$ ) if it is fundamental and if, further, the field of constants of $F\left\langle\eta_{1}, \cdots, \eta_{n}\right\rangle$ is an algebraic extension of $C$. By a theorem of Kolchin [2, Theorem 2], a regular system of zeros $\left(\eta_{1}, \cdots, \eta_{n}\right)$ of $L(y)$ always exists; the field of constants $D$ of $F\left\langle\eta_{1}, \cdots, \eta_{n}\right\rangle$ is then easily seen to be of finite degree over $C$. Our main result is to sharpen this existence theorem by proving the existence of a regular system of zeros $\left(\eta_{1}, \cdots, \eta_{n}\right)$ of $L(y)$ such that $D$ is a normal extension of $C$. When $\left(\eta_{1}, \cdots, \eta_{n}\right)$ has this property then every isomorphic image of $F\left\langle\eta_{1}, \cdots, \eta_{n}\right\rangle$ over $F$ has the same field of constants $D$.

It would be of interest to know whether $\left(\eta_{1}, \cdots, \eta_{n}\right)$ can be chosen so that $D=C$.

Preliminary lemmas. The paper consists essentially of two propositions which together comprise the existence theorem. For these we need several lemmas.

Lemma 1. Let $k$ be a constant. Then the field of constants of $F\langle k\rangle$ is $C(k)$.

This is proved in [3, Chapter I, Corollary 5 of Proposition 3].

Lemma 2. Suppose $0 \neq a \in F$. Then:

(1) if $\alpha$ is an integral of a (i.e. $\alpha^{\prime}=a$ ) transcendental over $F$, but the field of constants of $F\langle\alpha\rangle$ is algebraic over $C$, then that field of constants is $C$;

(2) if there exists an integral $\alpha$ of a algebraic over $F$ then there exists an integral of $a$ in $F$;

(3) Let $K$ be any algebraic extension of $C$ of finite degree over $C$. If there exists an integral $\alpha$ of a algebraic over $F$ then there exists an integral $\beta$ of a such that the field of constants of $F\langle\beta\rangle$ is $K$.

Proof. (1) follows from noting that $F\langle\alpha\rangle=F(\alpha)$ is a pure trans- 
cendental extension of $F$. To prove (2) suppose $\alpha$ is algebraic over $F$ and $\alpha^{\prime}=a$. If $\alpha \notin F$ let $\sum_{i=0}^{r} f_{i} X^{r-i}\left(r>1, f_{0}=1\right)$ be the irreducible polynomial over $F$ with root $\alpha$. Then $\sum_{i=0}^{r} f_{i} \alpha^{r-i}=0$ so

$$
\sum_{i=0}^{r}\left(f_{i}^{\prime} \alpha^{r-i}+a f_{i}(r-i) \alpha^{r-i-1}\right)=[0 .
$$

Since $f_{0}=1$ the left side of this equality is a polynomial in $\alpha$ over $F$ of degree at most $r-1$, hence it vanishes identically. In particular, since the coefficient of $\alpha^{r-1}$ vanishes we obtain $f_{1}^{\prime}+a r=0$. Hence $\beta=-f_{1} / r \in F$ is an integral of $a$, which proves (2). To prove (3), note first that there exists a constant $k$ such that $K=C(k)$. By (2) we may assume that $\alpha \in F$. Then $\beta=\alpha+k$ satisfies the conditions of (3). Q.E.D.

Corollary. Suppose $0 \neq a \in F$. Then there exists an integral $\alpha$ of a such that the field of constants of $F\langle\alpha\rangle$ is $C$.

Lemma 3. Suppose $0 \neq a \in F$ and let $P(y)=y^{\prime}-a y$. Then:

(1) if $\alpha$ is a nontrivial zero of $P(y)$ which is transcendental over $F$ but the field of constants of $F\langle\alpha\rangle$ is algebraic over $C$, then that field of constants is $C$;

(2) suppose there exists a nontrivial zero of $P(y)$ which is algebraic over $F$. Then any nontrivial algebraic zero $\alpha$ of $P(y)$ of minimal degree $r$ over $F$ has the property that $\alpha^{r} \in F$ and the field of constants of $F\langle\alpha\rangle$ is $C$.

Proof. (1) follows from the fact that $F\langle\alpha\rangle=F(\alpha)$. To prove (2) let $\alpha$ be a nontrivial algebraic zero of $P(y)$ of minimal degree $r$ over $F$. Let $\sum_{i=0}^{r} f_{i} X^{r-i}\left(r \geqq 1, f_{0}=1\right)$ be the irreducible polynomial over $F$ with root $\alpha$. Then $\sum_{i=0}^{r} f_{i} \alpha^{r-i}=0$. Differentiating this relation and utilizing the fact that $\alpha^{\prime}=a \alpha$ we obtain

$$
\sum_{i=0}^{r}\left(f_{i}^{\prime}+i a(r-i) f_{i}\right) \alpha^{r-i}=0 .
$$

Thus $\alpha$ is a root of the nonzero polynomial

$$
\sum_{i=0}^{r}\left(f_{i}^{\prime}+a(r-i) f_{i}\right) X^{r-i}
$$

over $F$. This latter polynomial is then a multiple of the former. Since both polynomials are of the same degree the factor is the coefficient $a r f_{0}=a r$ of $X^{r}$ in the second polynomial. Hence for $1 \leqq i \leqq r-1$ we have $f_{i}^{\prime}+a(r-i) f_{i}=a r f_{i}$ or $f_{i}^{\prime}=a i f_{i}$. If $f_{i}$ were different from zero for $1 \leqq i \leqq r-1$ then $f_{i}^{1 / t}$ would be a nontrivial zero of $P(y)$ algebraic over $F$ of degree $\leqq i<r$. This is impossible so $f_{1}=\cdots=f_{r-1}=0$. Hence 
$\alpha^{r}+f_{r}=0$ and $\alpha^{r} \in F$.

Now let $d \in F\langle\alpha\rangle$ be a constant. Then we can write $d=\sum_{i=0}^{r-1} g_{i} \alpha^{i}$ with each $g_{i} \in F$. Then, as above, $0=\sum_{i=0}^{r-1}\left(g_{i}^{\prime}+a i g_{i}\right) \alpha^{i}$. Equating to zero the coefficient of each $\alpha^{i}$ we obtain $g_{0}^{\prime}=0$ so $g_{0} \in C$ and for $i>0$ $g_{i}^{\prime}=-a i g_{i}$. If $g_{i}$ were different from zero for $i>0$ then $g_{i}^{-1 / 4}$ would be a nontrivial zero of $P(y)$ algebraic over $F$ of degree $\leqq i<r$. Hence $g_{1}=\cdots=g_{r-1}=0$ and $d=g_{0} \in C$. Q.E.D.

LemMa 4. Let $\left(\eta_{1}, \cdots, \eta_{n}\right)$ be a fixed regular system of zeros of $L(y)$. Let $\left(\zeta_{1}, \cdots, \zeta_{n}\right)$ be another regular system of zeros of $L(y)$ and let the field of constants of $F\left\langle\zeta_{1}, \cdots, \zeta_{n}\right\rangle$ be $E$. Then there exists a regular system of zeros $\left(\bar{\zeta}_{1}, \cdots, \bar{\zeta}_{n}\right)$ of $L(y)$ such that the field of constants $E_{1}$ of $F\left\langle\bar{\zeta}_{1}, \cdots, \bar{\zeta}_{n}\right\rangle$ contains $E$ and the field of constants of $F\left\langle\eta_{1}, \cdots, \eta_{n}\right.$, $\left.\bar{\zeta}_{1}, \cdots, \bar{\zeta}_{n}\right\rangle$ is an algebraic extension of $C$.

Proof. There exist constants $a_{i j}$ for $1 \leqq i, j \leqq n$ such that det $\left(a_{i j}\right)$ $\neq 0$ and $\zeta_{j}=\sum_{i=1}^{n} a_{i j} \eta_{i}(1 \leqq j \leqq n)$. Now it is easy to see that $F\left\langle\eta_{1}, \cdots\right.$, $\left.\eta_{n}, \zeta_{1}, \cdots, \zeta_{n}\right\rangle=F\left\langle\eta_{1}, \cdots, \eta_{n}, \cdots, a_{i j}, \cdots\right\rangle$. Hence if the field of constants of $F\left\langle\eta_{1}, \cdots, \eta_{n}\right\rangle$ is $D$, then by Lemma 1 the field of constants of $F\left\langle\eta_{1}, \cdots, \eta_{n}, \zeta_{1}, \cdots, \zeta_{n}\right\rangle$ is $D\left(\cdots, a_{i j}, \cdots\right)$. Therefore if each $a_{i j}$ is algebraic over $C$ we are finished.

Suppose then that some of the $a_{i j}$ are transcendental over $C$ hence over $C(D, E)$. There is a constant $e \in E$ such that $E=C(e)$ and we can write $e=f\left(\zeta_{1}, \cdots, \zeta_{n}\right) / g\left(\zeta_{1}, \cdots, \zeta_{n}\right)$ where $f\left(y_{1}, \cdots, y_{n}\right)$, $g\left(y_{1}, \cdots, y_{n}\right) \in F\left\{y_{1}, \cdots, y_{n}\right\}$.

Suppose the matrix $\left(a_{i j}\right)$ is specialized over $C(D, E)$ (hence over $\left.F(E)\left\langle\eta_{1}, \cdots, \eta_{n}\right\rangle\right)$ to a matrix $\left(\bar{a}_{i j}\right)$ of constants. Let $\bar{\zeta}_{j}=\sum_{i=1}^{n} \bar{a}_{i j} \eta_{i}$ $(1 \leqq j \leqq n)$. Then if $h\left(y_{1}, \cdots, y_{n}\right) \in F(E)\left\{y_{1}, \cdots, y_{n}\right\}$ is such that $h\left(\zeta_{1}, \cdots, \zeta_{n}\right)=0$, we have

$$
h\left(\sum_{i=1}^{n} a_{i 1} \eta_{i}, \cdots, \sum_{i=1}^{n} a_{i n} \eta_{i}\right)=0,
$$

so

$$
h\left(\sum_{i=1}^{n} \bar{a}_{i 1} \eta_{i}, \cdots, \sum_{i=1}^{n} \bar{a}_{i n} \eta_{i}\right)=0,
$$

whence $h\left(\bar{\zeta}_{1}, \cdots, \bar{\zeta}_{n}\right)=0$. Thus the family $\left(\bar{\zeta}_{j}^{(o)}\right)$ for $1 \leqq j \leqq n$ and $v=1,2, \cdots$ is a specialization over $F(E)$ of the family $\left(\zeta_{j}^{(0)}\right)$.

Since $\operatorname{det}\left(a_{i j}\right) \neq 0$ and $g\left(\zeta_{1}, \cdots, \zeta_{n}\right) \neq 0$ we can specialize the matrix $\left(a_{i j}\right)$ over $C(D, E)$ to a matrix $\left(\bar{a}_{i j}\right)$ of constants algebraic over $C$ such that det $\left(\bar{a}_{i j}\right) \neq 0$ and $g\left(\bar{\zeta}_{1}, \cdots, \bar{\zeta}_{n}\right) \neq 0$. Then $\left(\bar{\zeta}_{1}, \cdots, \bar{\zeta}_{n}\right)$ is a fundamental system of zeros of $L(y)$. Let $E_{1}$ be the 
field of constants of $F\left\langle\xi_{1}, \cdots, \bar{\zeta}_{n}\right\rangle$. Since the field of constants $D\left(\cdots, \bar{a}_{i j}, \cdots\right)$ of $F\left\langle\eta_{1}, \cdots, \eta_{n}, \bar{\zeta}_{1}, \cdots, \bar{\xi}_{n}\right\rangle$ is algebraic over $C$, so is $E_{1}$. Further, the equality $e=f\left(\zeta_{1}, \cdots, \zeta_{n}\right) / g\left(\zeta_{1}, \cdots, \zeta_{n}\right)$ implies that also $e=f\left(\bar{\zeta}_{1}, \cdots, \bar{\zeta}_{n}\right) / g\left(\bar{\zeta}_{1}, \cdots, \bar{\zeta}_{n}\right)$, whence $E \subseteq E_{1}$. Q.E.D.

Lemma 5. Suppose $n>1$, and $L(y)$ is such that whenever $\left(\eta_{1}, \cdots, \eta_{n}\right)$ and $\left(\zeta_{1}, \cdots, \zeta_{n}\right)$ are two regular systems of zeros of $L(y)$ with $\eta_{i}=\zeta_{j}$ for some $i, j$, then the fields of constants of $F\left\langle\eta_{1}, \cdots, \eta_{n}\right\rangle$ and $F\left\langle\zeta_{1}, \cdots, \zeta_{n}\right\rangle$ are equal. Then if $\left(\eta_{1}, \cdots, \eta_{n}\right)$ and $\left(\zeta_{1}, \cdots, \zeta_{n}\right)$ are any two regular systems of zeros of $L(y)$ the fields of constants of $F\left\langle\eta_{1}, \cdots, \eta_{n}\right\rangle$ and $F\left\langle\zeta_{1}, \cdots, \zeta_{n}\right\rangle$ are equal.

Proof. Let $\left(\eta_{1}, \cdots, \eta_{n}\right)$ be a regular system of zeros of $L(y)$ and let the field of constants of $F\left\langle\eta_{1}, \cdots, \eta_{n}\right\rangle$ be $D$. Let $\left(\zeta_{1}, \cdots, \zeta_{n}\right)$ be another regular system of zeros of $L(y)$ and let the field of constants of $F\left\langle\zeta_{1}, \cdots, \zeta_{n}\right\rangle$ be $E$. By Lemma 4 choose a regular system of zeros $\left(\bar{\zeta}_{1}, \cdots, \bar{\zeta}_{n}\right)$ of $L(y)$ such that the field of constants of $F\left\langle\eta_{1}, \cdots, \eta_{n}, \bar{\zeta}_{1}, \cdots, \bar{\zeta}_{n}\right\rangle$ is algebraic over $C$ and the field of constants $E_{1}$ of $F\left\langle\bar{\zeta}_{1}, \cdots, \bar{\zeta}_{n}\right\rangle$ contains $E$.

We can write $\bar{\zeta}_{j}=\sum_{i=1}^{n} \bar{a}_{i j} \eta_{i}(1 \leqq j \leqq n)$ where the $\bar{a}_{i j}$ are constants algebraic over $C$ and $\operatorname{det}\left(\bar{a}_{i j}\right) \neq 0$. Therefore we can choose one of the $\eta_{i}$ so that the set $\left(\eta_{i}, \bar{\zeta}_{2}, \cdots, \bar{\zeta}_{n}\right)$ is linearly independent over constants. Then this set is a regular system of zeros of $L(y)$ so the field of constants of $F\left\langle\eta_{i}, \bar{\zeta}_{2}, \cdots, \bar{\zeta}_{n}\right\rangle$ is $D$. Then the field of constants of $F\left\langle\bar{\zeta}_{1}, \cdots, \bar{\zeta}_{n}\right\rangle$ is $D$. Thus $D=E_{1} \supseteq E$.

By reversing the roles of $\left(\eta_{1}, \cdots, \eta_{n}\right)$ and $\left(\zeta_{1}, \cdots, \zeta_{n}\right)$ we obtain $E \supseteq D$. Hence $D=E$. Q.E.D.

Lemma 6. Let $p_{0}=1$. Let $\eta$ be a nontrivial zero of $L(y)$ and let

$$
M(t)=\sum_{r=0}^{n-1} t^{(r)} \sum_{k=r+1}^{n} p_{n-k}\left(\begin{array}{c}
k \\
r+1
\end{array}\right) \eta^{(k-r-1)} .
$$

Then $M(t)$ is a homogeneous linear ordinary differential polynomial over $F\langle\eta\rangle$ different from zero and of order $n-1$. Let $\left(\zeta_{1}, \cdots, \zeta_{n-1}\right)$ be any fundamental system of zeros of $M(t)$ and let $\mu_{1}, \cdots, \mu_{n-1}$ be such that $\mu_{i}^{\prime}=\zeta_{i}$ for $1 \leqq i \leqq n-1$. Then $\left(\eta, \eta \mu_{1}, \cdots, \eta \mu_{n-1}\right)$ is a fundamental system of zeros of $L(y)$.

Proof. This is well known and the proof is classical.

The main result. We now prove the two propositions leading to the existence theorem.

PROPOSITION 1. Suppose there exist two regular systems of zeros of 
$L(y),\left(\eta_{1}, \cdots, \eta_{n}\right)$ and $\left(\zeta_{1}, \cdots, \zeta_{n}\right)$, such that the fields of constants of $F\left\langle\eta_{1}, \cdots, \eta_{n}\right\rangle$ and $F\left\langle\zeta_{1}, \cdots, \zeta_{n}\right\rangle$ are unequal. Let $K$ be a subfield of $C$ with $[C: K]$ finite. Then there exists a regular system of zeros $\left(\mu_{1}, \cdots, \mu_{n}\right)$ of $L(y)$ such that the field of constants of $F\left\langle\mu_{1}, \cdots, \mu_{n}\right\rangle$ is normal over $K$.

Proof. We prove this by induction on $n$. For $n=1$ write $L(y)=y^{\prime}$ $-a y$. If $a=0$ the result is obvious so suppose $a \neq 0$. There exists a nontrivial zero $\alpha$ of $L(y)$ such that the field of constants of $F\langle\alpha\rangle$ is algebraic over but not equal to $C$. Hence by Lemma 3, $\alpha$ is algebraic over $F$ so there is an element $\beta \neq 0$ algebraic over $F$ satisfying:

(1) $\beta^{\prime}-a \beta=0$;

(2) of all nontrivial zeros of $L(y)$ algebraic over $F, \beta$ is of minimal degree $r$ over $F$ and $b=\beta^{r} \in F$;

(3) the field of constants of $F\langle\beta\rangle$ is $C$.

Let $K_{1}$ be a normal extension of $K$ of finite degree over $K$ and containing $C$. There is a constant $k$ such that $K_{1}=C(k)$. Let $u^{r}=k$ and let $\gamma=u \beta$. Then $\gamma$ is a zero of $L(y)$ and $\gamma^{n}=k b$ so the field of constants of $F\langle\gamma\rangle$ contains $K_{1}$.

Now $[F(k)\langle\gamma\rangle: F(k)] \leqq r$. Suppose there is a nontrivial zero $\delta$ of $L(y)$ such that $[F(k)\langle\delta\rangle: F(k)]=s<r$. By Lemma 3 we can choose $s$ minimal so $\delta^{s} \in F(k)$. Then we can write $\delta^{s}=\sum_{i=0}^{q} f_{i} k^{i}$ where each $f_{i} \in F$ and $1, k, \cdots, k^{q}$ are linearly independent over $F$. Then $s \delta^{s-1} \delta^{\prime}$ $=\sum_{i=0}^{q} f_{i}^{\prime} k^{i}$ or $s a \delta^{s}=\sum_{i=0}^{q} f_{i}^{\prime} k^{i}$. Hence for $0 \leqq i \leqq q$ we have $f_{i}^{\prime}=s a f_{i}$. If $f_{i}$ were different from zero then $f_{l}^{1 / s}$ would be a nontrivial zero of $L(y)$ of degree $\leqq s<r$ over $F$. This is impossible so $[F(k)\langle\gamma\rangle: F(k)]=r$. Thus $\gamma$ is a nontrivial zero of $L(y)$ of minimal degree over $F(k)$. Hence by Lemma 3 the field of constants of $F\langle\gamma\rangle$ is equal to the field of constants of $F(k)$ which is $C(k)=K_{1}$ by Lemma 1 .

Now suppose $n \geqq 2$ and that the proposition is true for $n-1$. By Lemma 5 there exist two regular systems of zeros of $L(y)$, $\left(\eta, \mu_{1}, \cdots, \mu_{n-1}\right)$ and $\left(\eta, \zeta_{1}, \cdots, \zeta_{n-1}\right)$, such that the field of constants $D$ and $E$ of $F\left\langle\eta, \mu_{1}, \cdots, \mu_{n-1}\right\rangle$ and $F\left\langle\eta, \zeta_{1}, \cdots, \zeta_{n-1}\right\rangle$ respectively are unequal. Let $p_{0}=1$ and let

$$
M(t)=\sum_{j=0}^{n-1} t^{(j)} \sum_{i=j+1}^{n} p_{n-i}\left(\begin{array}{c}
i \\
j+1
\end{array}\right) \eta^{(i-j-1)} .
$$

It is easily seen that $\left(\left(\mu_{1} / \eta\right)^{\prime}, \cdots,\left(\mu_{n-1} / \eta\right)^{\prime}\right)$ and $\left(\left(\zeta_{1} / \eta\right)^{\prime}, \cdots\right.$, $\left.\left(\zeta_{n-1} / \eta\right)^{\prime}\right)$ are two regular systems of zeros of $M(t)$. Let $G_{1}$ $=F\left\langle\eta,\left(\mu_{1} / \eta\right)^{\prime}, \cdots,\left(\mu_{n-1} / \eta\right)^{\prime}\right\rangle$ and $G_{2}=F\left\langle\eta,\left(\zeta_{1} / \eta\right)^{\prime}, \cdots,\left(\zeta_{n-1} / \eta\right)^{\prime}\right\rangle$. We consider two possibilities:

(1) the fields of constants of $G_{1}$ and $G_{2}$ are equal; 
(2) the fields of constants of $G_{1}$ and $G_{2}$ are unequal.

In case (1) either the field of constants of $G_{1}$ is not $D$ or the field of constants of $G_{2}$ is not $E$. Suppose the former. Now $F\left\langle\eta, \mu_{1}, \cdots\right.$, $\left.\mu_{n-1}\right\rangle$ is obtained from $G_{1}$ by the successive adjunctions of the integrals $\left(\mu_{1} / \eta\right), \cdots,\left(\mu_{n-1} / \eta\right)$ of elements of $G_{1}$. Therefore by Lemma 2 , for some $1 \leqq i \leqq n-1,\left(\mu_{i} / \eta\right)$ is algebraic over $G_{1}\left\langle\left(\mu_{1} / \eta\right), \cdots,\left(\mu_{i-1} / \eta\right)\right\rangle$. Choose such a value of $i$. Let $\lambda_{1}=\mu_{1} / \eta, \cdots, \lambda_{i-1}=\mu_{i-1} / \eta$, and let $D_{1}$ be the field of constants of $G_{1}\left\langle\lambda_{1}, \cdots, \lambda_{i-1}\right\rangle$. Then $D_{1}$ is an algebraic extension of finite degree over $C$ hence over $K$. Let $K_{1}$ be a normal extension of finite degree over $K$ which contains $D_{1}$. By Lemma 2 we can find an integral $\lambda_{i}$ of $\left(\mu_{i} / \eta\right)^{\prime}$ such that the field of constants of $G_{1}\left\langle\lambda_{1}, \cdots, \lambda_{i-1}, \lambda_{i}\right\rangle$ is $K_{1}$. And by the corollary to Lemma 2 we can find integrals $\lambda_{i+1}, \cdots, \lambda_{n-1}$ of $\left(\mu_{i+1} / \eta\right)^{\prime}, \cdots,\left(\mu_{n-1} / \eta\right)^{\prime}$ respectively such that the field of constants of $G_{1}\left\langle\lambda_{1}, \cdots, \lambda_{n-1}\right\rangle$ is $K_{1}$. But we have

$$
\begin{aligned}
G_{1}\left\langle\lambda_{1}, \cdots, \lambda_{n-1}\right\rangle & =F\left\langle\eta,\left(\mu_{1} / \eta\right)^{\prime}, \cdots,\left(\mu_{n-1} / \eta\right)^{\prime}\right\rangle\left\langle\lambda_{1}, \cdots, \lambda_{n-1}\right\rangle \\
& =F\left\langle\eta, \lambda_{1}, \cdots, \lambda_{n-1}\right\rangle \\
& =F\left\langle\eta, \eta \lambda_{1}, \cdots, \eta \lambda_{n-1}\right\rangle .
\end{aligned}
$$

By Lemma $6,\left(\eta, \eta \lambda_{1}, \cdots, \eta \lambda_{n-1}\right)$ is a fundamental system of zeros of $L(y)$ which proves the proposition in case (1).

If case (2) applies, denote by $C_{1}$ the field of constants of $F\langle\eta\rangle$. Then $\left[C_{1}: K\right]$ is finite and $M(t)$ satisfies the hypotheses of the proposition, so by the induction hypothesis there is a regular system of zeros $\left(\xi_{1}, \cdots, \xi_{n-1}\right)$ of $M(t)$ such that the field of constants of $F\left\langle\eta, \xi_{1}, \cdots, \xi_{n-1}\right\rangle$ is a normal algebraic extension of $K$. By the corollary to Lemma 2 we can find integrals $\theta_{1}, \ldots, \theta_{n-1}$ of $\xi_{1}, \cdots, \xi_{n-1}$ respectively such that the field of constants of $F\left\langle\eta, \xi_{1}, \cdots, \xi_{n-1}\right\rangle$ $\left\langle\theta_{1}, \cdots, \theta_{n-1}\right\rangle$ is equal to the field of constants of $F\left\langle\eta, \xi_{1}, \cdots, \xi_{n-1}\right\rangle$, hence is normal over $K$. But $F\left\langle\eta, \xi_{1}, \cdots, \xi_{n-1}\right\rangle\left\langle\theta_{1}, \cdots, \theta_{n-1}\right\rangle$ $=F\left\langle\eta, \eta \theta_{1}, \cdots, \eta \theta_{n-1}\right\rangle$ so by Lemma 6 the proposition is proved. Q.E.D.

Lemma 7. Suppose there exists a field $D$ such that for every fundamental system of zeros $\left(\eta_{1}, \cdots, \eta_{n}\right)$ of $L(y)$ the field of constants of $F\left\langle\eta_{1}, \cdots, \eta_{n}\right\rangle$ is $D$. Then $D=C$.

Proof. Since $D$ is of finite degree over $C$ there is an element $d \in D$ such that $D=C(d)$. Let $\left(\eta_{1}, \cdots, \eta_{n}\right)$ be any fundamental system of zeros of $L(y)$ and let $\left(x_{i j}\right)$ for $1 \leqq i, j \leqq n$ be constants algebraically independent over $F\left\langle\eta_{1}, \cdots, \eta_{n}\right\rangle$. Let $\mu_{j}=\sum_{i=1}^{n} x_{i j} \eta_{i}(1 \leqq j \leqq n)$. Then $\left(\mu_{1}, \cdots, \mu_{n}\right)$ is a fundamental system of zeros of $L(y)$ so there 
exist $f\left(y_{1}, \cdots, y_{n}\right), g\left(y_{1}, \cdots, y_{n}\right) \in F\left\{y_{1}, \cdots, y_{n}\right\}$ such that $g\left(\mu_{1}, \cdots, \mu_{n}\right) \neq 0$ and $d=f\left(\mu_{1}, \cdots, \mu_{n}\right) / g\left(\mu_{1}, \cdots, \mu_{n}\right)$. Clearly we may assume that $f\left(y_{1}, \cdots, y_{n}\right)$ and $g\left(y_{1}, \cdots, y_{n}\right)$ are of order $\leqq n-1$ in each $y_{i}$. Further, we choose $g\left(y_{1}, \cdots, y_{n}\right)$ so that one of its coefficients is equal to 1 . Then

$$
g\left(\sum_{i=1}^{n} x_{i 1} \eta_{i}, \cdots, \sum_{i=1}^{n} x_{i n} \eta_{i}\right) d-f\left(\sum_{i=1}^{n} x_{i 1} \eta_{i}, \cdots, \sum_{i=1}^{n} x_{i n} \eta_{i}\right)=0 .
$$

This is an identity in the elements $x_{i j}$. Let $\zeta_{1}, \cdots, \zeta_{n}$ be any $n$ zeros of $L(y)$. Then there are constants $a_{i j}(1 \leqq i, j \leqq n)$ such that $\zeta_{j}$ $=\sum_{i=1}^{n} a_{i j} \eta_{i}(1 \leqq j \leqq n)$. Then we have

$$
g\left(\sum_{i=1}^{n} a_{i 1} \eta_{i}, \cdots, \sum_{i=1}^{n} a_{i n} \eta_{i}\right) d-f\left(\sum_{i=1}^{n} a_{i 1} \eta_{i}, \cdots, \sum_{i=1}^{n} a_{i n} \eta_{i}\right)=0
$$

or $g\left(\zeta_{1}, \cdots, \zeta_{n}\right) d-f\left(\zeta_{1}, \cdots, \zeta_{n}\right)=0$. Thus the differential polynomial $g\left(y_{1}, \cdots, y_{n}\right) d-f\left(y_{1}, \cdots, y_{n}\right) \in F(d)\left\{y_{1}, \cdots, y_{n}\right\}$ vanishes for every zero of the perfect differential ideal $\left\{L\left(y_{1}\right), \cdots, L\left(y_{n}\right)\right\}$ $\subseteq F(d)\left\{y_{1}, \cdots, y_{n}\right\}$. Hence [4, Chapter 2, $\left.\$ 7\right] g\left(y_{1}, \cdots, y_{n}\right) d$ $-f\left(y_{1}, \cdots, y_{n}\right)$ is an element of that perfect differential ideal. It is easy to see, however, that any nonzero element of $\left\{L\left(y_{1}\right), \cdots, L\left(y_{n}\right)\right\}$ has order $\geqq n$ in at least one $y_{i}$. Therefore $g\left(y_{1}, \cdots, y_{n}\right) d$ $-f\left(y_{1}, \cdots, y_{n}\right)=0$. But one of the coefficients in $g\left(y_{1}, \cdots, y_{n}\right)$ is equal to 1 , so one coefficient in $f\left(y_{1}, \cdots, y_{n}\right)$ is $d$. Thus $d \in F$. Q.E.D.

Proposition 2. Suppose there exists a field $D$ such that for every regular system of zeros $\left(\eta_{1}, \cdots, \eta_{n}\right)$ of $L(y)$ the field of constants of $F\left\langle\eta_{1}, \cdots, \eta_{n}\right\rangle$ is $D$. Then either $D=C$ or $D=\bar{C}$.

PROOF. If for every fundamental system of zeros $\left(\mu_{1}, \cdots, \mu_{n}\right)$ of $L(y)$ the field of constants of $F\left\langle\mu_{1}, \cdots, \mu_{n}\right\rangle$ is $D$, then, by Lemma 7 , $D=C$. Suppose then that for some fundamental system of zeros $\left(\zeta_{1}, \cdots, \zeta_{n}\right)$ of $L(y)$ the field of constants of $F\left\langle\zeta_{1}, \cdots, \zeta_{n}\right\rangle$ is not $D$. Suppose also that $D \neq \bar{C}$.

Now the field of constants $E$ of $F\left\langle\zeta_{1}, \cdots, \zeta_{n}\right\rangle$ is transcendental over $C$ so we may write $E=C\left(\alpha_{1}, \cdots, \alpha_{s}\right)$ where $\alpha_{1} \cdots, \alpha_{r}(r \geqq 1)$ is a transcendence base for $E$ over $C$. Then there are differential polynomials $f\left(y_{1}, \cdots, y_{n}\right), g\left(y_{1}, \cdots, y_{n}\right) \in F\left\{y_{1}, \cdots, y_{n}\right\}$ such that $g\left(\zeta_{1}, \cdots, \zeta_{n}\right) \neq 0$ and $\alpha_{1}=f\left(\zeta_{1}, \cdots, \zeta_{n}\right) / g\left(\zeta_{1}, \cdots, \zeta_{n}\right)$. Let $\left(\eta_{1}, \cdots, \eta_{n}\right)$ be any regular system of zeros of $L(y)$. Then there are constants $\beta_{i j}(1 \leqq i, j \leqq n)$ such that $\operatorname{det}\left(\beta_{i j}\right) \neq 0$ and $\zeta_{j}=\sum_{i=1}^{n} \beta_{i j} \eta_{i}(1 \leqq j \leqq n)$. Thus 


$$
g\left(\sum_{i=1}^{n} \beta_{i 1} \eta_{i}, \cdots, \sum_{i=1}^{n} \beta_{i n} \eta_{i}\right) \alpha_{1}-f\left(\sum_{i=1}^{n} \beta_{i 1} \eta_{i}, \cdots, \sum_{i=1}^{n} \beta_{i n} \eta_{i}\right)=0 .
$$

There exists a polynomial $P(X) \in D[X]$ such that any specialization of $\alpha_{1}$ to a constant $a_{1}$ over $D$ (hence over $F\left\langle\eta_{1}, \cdots, \eta_{n}\right\rangle$ ) such that $P\left(a_{1}\right) \neq 0$ can be extended to a specialization $\left(a_{1}, \cdots\right.$, $\left.a_{s}, \cdots, b_{i j}, \cdots\right)$ over $D$ of $\left(\alpha_{1}, \cdots, \alpha_{s}, \cdots, \beta_{i j}, \cdots\right)$ such that $g\left(\sum_{i=1}^{n} b_{i 1} \eta_{i}, \cdots, \sum_{i=1}^{n} b_{i n} \eta_{i}\right) \neq 0$ and $\operatorname{det}\left(b_{i j}\right) \neq 0$. Since $D \neq \bar{C}$ so that $\bar{C}-D$ is infinite and since $\alpha_{1}$ is transcendental over $D$ we can specialize $\alpha_{1}$ to an element $a_{1} \in \bar{C}-D$ such that $P\left(a_{1}\right) \neq 0$. We can then extend this to the specialization above with the stated properties. Further, we may choose each $a_{i}, b_{i j} \in \bar{C}$.

Let $\bar{\zeta}_{j}=\sum_{i=1}^{n} b_{i j} \eta_{i}(1 \leqq j \leqq n)$. Then the field of constants of $F\left\langle\eta_{1}, \cdots, \eta_{n}, \bar{\zeta}_{1}, \cdots, \bar{\zeta}_{n}\right\rangle$ is $D\left(\cdots, b_{i j}, \cdots\right)$ so $\left(\bar{\zeta}_{1}, \cdots, \bar{\xi}_{n}\right)$ is a regular system of zeros of $L(y)$. On the other hand, we have $g\left(\bar{\zeta}_{1}, \cdots, \bar{\zeta}_{n}\right) a_{1}=f\left(\bar{\zeta}_{1}, \cdots, \bar{\zeta}_{n}\right)$ so $a_{1} \in F\left\langle\bar{\zeta}_{1}, \cdots, \bar{\zeta}_{n}\right\rangle$. Hence the field of constants of $F\left\langle\bar{\zeta}_{1}, \cdots, \bar{\zeta}_{n}\right\rangle$ is not $D$. This is a contradiction. Q.E.D.

THEOREM. There exists a fundamental system of zeros $\left(\eta_{1}, \cdots, \eta_{n}\right)$ of $L(y)$ such that the field of constants of $F\left\langle\eta_{1}, \cdots, \eta_{n}\right\rangle$ is a normal algebraic extension of $C$.

Proof. This follows from Propositions 1 and 2.

\section{BIBLIOGRAPHY}

1. E. R. Kolchin, Algebraic matric groups and the Picard-Vessiot theory of homogeneous linear ordinary differential equations, Ann. of Math. (2) vol. 49 (1948) pp. $1-42$.

2. - Existence theorems connected with the Picard-Vessiot theory of homogeneous linear ordinary differential equations, Bull. Amer. Math. Soc. vol. 54 (1948) pp. 927-932.

3. - Galois theory of differential fields, Amer. J. Math. vol. 75 (1953) pp. 753-824.

4. J. F. Ritt, Differential algebra, Amer. Math. Soc. Colloquium Publications, vol. 33, 1950.

5. A. Weil, Foundations of algebraic geometry, Amer. Math. Soc. Colloquium Publications, vol. 29, 1946.

Columbia University aNd

University of California, Berkeley 